\title{
Sitzung vom 26. Juli 1880.
}

Vorsitzender: Hr. A. W. H o fman n, Vice-Präsident.

Das Protocoll der letzten Sitzung wird genehmigt.

Zu ausserordentlichen Mitgliedern werden proclamirt die Herren:

Hugo Vosswinkel,

Theodor Lupp,

Anton Heyroth, $\quad$ Berlin;

Friedrich Bloem,

Dr. E. Simon,

J. P. Venable, Bonn;

Alexander K. Miller, Würzburg;

Dr. Otto Mittenzwey, Erlangen.

$\mathrm{Za}$ ausserordentlichen Mitgliedern werden vorgeschlagen die Herren :

Georg Grund, JPhysiol. chem. Laborat. Leipzig (durch

Max Pröpper, des Hofrath Wiede- A. Keil und

Eugen Waas, mann,

Reinhard Wagner, Dresdenerstr. 20 I.,

C. Councler);

Dr. K. Böhmer, Assist. d. agriculturchem. Versuchs-Station, Münster i./W. (durch A. Hilger und J. Volbard);

Dr. Paul Degener, Laborator. des Vereins für RübenzuckerIndustrie,

Dr. H. Troschke, Laborat. des landwirthschaftl. Instituts,

Berlin N., Invalidenstrasse $42 / 47$ (durch H. Landolt and

F. Tiemann).

Für die Bibliothek sind als Geschenke eingegangen:

867. Schmidt, Carl. Chemische Untersuchung der Ackerkrume und des Untergrundes aus 8 Arten der Schwarzerderegion und ihres Grenzgebietes. Dorpat 1880. Sep.-Abdr. (Verf.)

Berichte d. D. chen. Gesellschaft. Jahrg. XIII. 
1039. Berger, Franz. Zur Kenntniss guanidiuartiger Verbindungen. Inaug.Diss. Berlin 1880. (Verf.)

1040. Hehner, Otto. Alcohol tables. London 1880. (Verf.)

1041. Möller, Hermann. Die Cyanamidverbindungen der Bernsteinsäure. Inaug.Diss. Leipzig 1880. (Verf.)

1042. Spring, Walthère. Recherches sur la propriété que possèdent les corps de se souder sous l'action de la pression. Bruxelles 1880. Sep.-Abdr. (Verf.)

1043. Deutsch, Alfred. Ueber schwefelhaltige Derivate des Diphenyls. Beiträge zur Kenntniss der Dicyanamidobenzoësäure und der Aether der Orthoameisensäure. Inaug.-Diss. Berlin 1880. (Verf.)

1044. Czyrniański, Emil. O przyciąganin jako objarie dopelniczym rucbu chemicznego. W Krakowie 1880. (Verf.)

66. Polytechnisches Notizblatt 1880. Nos 13 und 14. (Vom Herausgeber.)

363. Kopp, Hermann. Aurea catena Homeri. Braunschweig 1880.

Im Anschluss an die Mittheilung des Hrn. Bibliothekars bemerkt der Vorsitzende, dass er sich glücklich schätze, noch ein weiteres Geschenk auzeigen zu können, welches, er zweifle nicht daran, die Gesellschaft aus mehr als einem Grunde in freudiger Dankbarkeit entgegennebmen werde. Es sei dies eine Schrift unseres Hrn. Präsidenten, welche er im Auftrage desselben überreiche. Das Interesse an dem Inbalte des Büchleins werde in diesem Falle noch besonders durch den Umstand erhöbt, dass dasselbe als Festschrift bestimmt sei, den am 31. d. M. stattfindenden Geburtstag Friedricb Wöhlers, an welchem unser Ebrenmitglied und früherer Präsident sein achtzigstes Lebensjahr zurücklegen wird, zu verherrlichen. Das Buch führe den Titel: A urea catena Homeri. Ueber den Inhalt desselben sage Hr. Kopp in der Zueignung an F. Wöhler anter Anderem folgendes:

${ }_{n}$ Die goldene Kette des Homer ist der Gegenstand des Angebindes, welches ich dir widme. Diese goldene Kette ist nicht ein Ergebniss von Ausgrabungeu, wie sie in neuerer Zeit mit so staunenswerthen Resultaten ausgeführt worden sind. Aber Etwas wie eine Ausgrabung gehört doch auch wesentlich mit zu dem, was das rorliegende Schriftchen etwa Nützliches bringen mag: dass in ihm an's Licht gezogen wird ein vor 134 Jahren begrabener Mann, der wohl auch bei Lebzeiten nur wenigen als Schriftsteller bekanut war, und dessen dann gar nicht mehr gedacht worden ist, und weleber doch durch ein Werk im vorigen Jahrbundert auf unzäblige, unter diesen auch auf Göthe in der Jugend desselben, erheblichen Einfluss ausgeübt hat, und hiernach, dass man sich seiner erinnere, mehr verdient, als Viele, deren Namen sich in der Literär-Geschichte erhalten haben."

Im Anschluss an diese Mittheilung lenkt der Vorsitzende die Aufmerksawkeit der Versammlung auf ein ron dem Bildhauer Hrn. Prof. Ed. Lürssen ausgestelltes schönes Marmorrelief Friedrich 
Wöhler's in pracbtroll gegliederter Bronzeumrabmung, sowie aut eine goldene Gedächtnissmedaille, Ehrengeschenke, welche Freunde, Schüler und Facbgenossen dem verebrten Meister am 31. Juli als Angebinde zu seinem Geburtstage überreichen werden.

An der Feier dieses Geburtstages will sich auch die Gesellschaft betheiligen, und der Vorsitzende wird beauftragt, ibrem Ebrenmitgliede die berzlichen Geburtstagswünsche der Vereinsgenossen in einem Festtelegramm auszusprechen.

Hr. Landolt glaubt eine die Mitglieder der Gesellschaft interessirende Angelegenheit zur Sprache zu bringen, indem er den Sekretär fragt, bis zu welchem Zeitpunkt die Veröffentlichung des mit Ungeduld erwarteten Registers über die ersten 10 Jahrgänge der Berichte entgegengesehen werden könne. Der Sekretär erwidert, dass Sach- und Autorenregister fertig gedruckt vorliegen, dass Herr Dr. Bisch of $f$ zur Zeit mit der Abfassung des systematischen Registers beschäftigt sei, und dass seitens des Sekretariats Schritte geschehen werden, um die Vollendung des Werkes bis zum Anfang des Wintersemesters zu ermöglichen.

Der Vorsitzende begrüsst das in der Sitzung anwesende auswärtige Mitglied der Gesellschaft, Herrn Dr. F. F i $8 \mathrm{cber}$ aus Hannover.

Der Schriftführer: Der Vorsitzende:
A. Pinner.
A. W. H of mang.

\section{Mittheilungen.}

\section{Richard Meyer und Albert Baur: Oeber Hydroxylirung durch direkte Oxydation.}

(Eingegangen am 12. Juli; verl. in der Sitzung von Hrn. A. Pinner.)

Im weiteren Verfolg unserer Untersuchungen ${ }^{1}$ ) haben wir nun auch das Verhalten des Cymols gegen übermangansaures Kalium geprüft. Da dieses nach zahlreichen Untersuchungen jetzt wohl 2 weifellos als normales Propyltoluol zu betrachten ist, so war es von vornberein nicht wahrscheinlich, dass es sich direkt in eine Oxyverbindung überfübren lassen würde; denn nach den bisherigen Erfabrungen gelingt dies nur bei solchen Körpern, welche tertiäre Wasserstoffatome enthalten.

1) Diese Berichte XI, 1283, 1787, 1790, 2172. XII, 1071, 2:38. 